\title{
Improving novel motor learning through prior high contextual interference training
}

\author{
T. Kim ${ }^{\mathrm{a}}$, J. Chen ${ }^{\mathrm{a}}$, W.B. Verwey ${ }^{\mathrm{b}, \mathrm{a}}$, D.L. Wright ${ }^{\mathrm{a}, *}$ \\ a Texas A\&M University, United States \\ ${ }^{\mathrm{b}}$ University of Twente, The Netherlands
}

\section{A R T I C L E I N F O}

\section{Keywords:}

Contextual interference

Sequence learning

Motor learning

Concatenation

Consolidation

\begin{abstract}
A B S T R A C T
The primary objective of the present experiment was to examine the influence of recent practice in a random and blocked format for future motor learning. First, individuals practiced three unique discrete sequence production tasks in either a blocked or random schedule. One day later, all individuals practiced a new motor sequence not previously practiced. On day three, mean total time for the test performance of the original three motor sequences was lower for individuals that practiced in a random format. This emerged as a significant reduction in mean total time from the completion of practice and the test trials implicating offline consolidation as a key contributor to the random practice performance advantage. A novel finding from the present work was that the acquisition of the novel discrete sequence production task practiced on Day 2 was better for individuals that had prior random rather than blocked practice experience. This benefit was robust appearing early during acquisition as significantly lower mean total time. This benefit from random practice experience remained during the delayed test trials administered on Day 3 for the novel motor sequence.
\end{abstract}

\section{Introduction}

The scheduling of practice that is most suited to facilitate the acquisition of multiple motor skills has been the subject of considerable experimental examination. One practice phenomenon focused on the best practice for learning multiple related skills is examined under the general rubric of the contextual interference (CI) effect (Brady, 2004; Magill \& Hall, 1990; Shea \& Morgan, 1979; Wright et al., 2016). This practice effect typically involves the comparison of the learning gains from random (RP) and blocked (BP) scheduling formats. On the one hand, RP is a relatively high interference practice environment because multiple motor skills are practiced concurrently thus demanding the learner to navigate constantly changing task demands across practice. In contrast, BP induces less interference throughout training because it involves the repeated performance of the same motor task for a predetermined number of trials before practice of other motor tasks. It turns out that RP, while characterized by relatively slow initial performance during training, is more effective for supporting long-term retention of the practiced skills. This finding, while frequently reported in the laboratory environment (Wright et al., 2016), has also emerged in various applied (Goode \& Magill, 1986; Ollis, Button, \& Fairweather, 2005; Schneider, Healy, \& Bourne, 1998; Smith \& Davies, 1995) and rehabilitative settings (Adams \& Page, 2000; Hanlon, 1996; Knock,
Ballard, Robin, \& Schmidt, 2000).

An important consequence of extensive physical practice is the emergence of transient functional connectivity and structural adaptation between and within neural networks to support skilled motor behavior (Dayan \& Cohen, 2011). Interestingly, it is now clear that the practice schedule to which the learner is exposed, as well as extent, plays a role in promoting inter-regional functional connectivity. For example, Lin et al. (2013) examined fMRI data collected during RP and $\mathrm{BP}$ and noted that RP, but not BP, led to a temporary coupling between dorsolateral prefrontal cortex (DLPFC) and premotor (PM) areas with key sensorimotor sites for up to 72-h after the completion of practice. Furthermore, as this connectivity developed there was a concomitant reduction in blood oxygenated level dependent signal at the neural sites involved which was interpreted as an increased efficiency and/or economy for planning learned behaviors via RP. Lin et al. (2013) claimed that a critical consequence of experiencing greater CI during practice was improvement in the communication between a frontal "strategic" network and the sensorimotor network to facilitate successful delayed retrieval of newly learned motor tasks (see also, Yang, $\mathrm{Li}$, \& Chiang, 2014). The notion that RP leads to the development of an extensive retrieval network is not new, first being noted in behavioral accounts, and is central to most descriptions of savings or retention benefits observed following high interference training (Lin et al., 2013;

\footnotetext{
* Corresponding author

E-mail address: davidwright@tamu.edu (D.L. Wright).
} 
Shea \& Zimny, 1983, 1988; Wright et al., 2016).

To date, enhanced learning from RP is manifest as superior execution of the specific skills that are included in the original bout of BP or RP during a delayed test often administered in a random schedule format. Fewer studies have adopted a blocked test format and revealed RP benefits (Wright, Brueckner, Black, Magnuson, \& Immink, 2004). Recently, a couple of studies broadened the scope of investigation of this practice phenomenon by considering the impact of recent high-CI practice on subsequent motor learning (Hodges, Lohse, Wilson, Lim, \& Mulligan, 2014; Kim, Rhee, \& Wright, 2016). The specific objective of these studies was to assess the importance of the learner's practice history, particularly BP or RP, for acquisition of novel motor skills. The basic premise of these efforts was that if RP results in the establishment of an extensive memory network from recent practice experience, future learning of related skills would benefit. This might emerge as faster initial encoding of new knowledge, reflected in improved performance in acquisition, and/or superior retention of a novel skill compared to BP counterparts.

An initial assessment of this issue by Hodges et al. (2014) evaluated the influence of BP or RP on the learning of three new motor skills practiced 24-hrs later in (a) either a blocked or random format, or (b) a self-selected practice schedule. While prior RP enhanced the acquisition of novel skills on Day 2, which meant the typical performance deficit associated with a high CI practice environment was eliminated, delayed retention for the new skills was not dependent on a learner's previous training history. Kim et al. (2016), using a similar design to Hodges et al., addressed this same question but simplified the new learning environment to the acquisition of just a single rather than multiple skills, following BP or RP. Again, experience with RP accelerated the encoding of the new skill but failed to offer any further benefit across the retention interval beyond that observed from BP. These data then verified those of Hodges et al. (2014) suggesting some limited utility of a recent history with high CI training for later periods of skill acquisition.

Kim et al. (2016) noted that one feature of their study that may have restricted the effectiveness of RP for new motor learning was the use of a serial reaction time (RT) task for which the learner completed a series of seven key-presses as frequently as possible during a 30-s trial (see, Walker, Brakefield, Hobson, \& Stickgold, 2003). In hindsight, the use of the serial RT task by Kim et al. may have inadvertently compromised the magnitude of CI created during RP because each 30-s trial involved the execution of 5-15 repetitions of the same motor sequence (i.e., essentially a form of BP). Thus, it is likely that the RP condition used by Kim et al. (2016) induced significantly lower CI than typically created in previous studies (Brady, 2004; Magill \& Hall, 1990; Wright et al., 2016). A primary goal of the present work then was to address this shortcoming and re-evaluate the potential robustness of prior high CI training for new motor learning. To accomplish this, the present work involved the practice of a number of discrete sequence production (DSP) tasks in either a RP or BP format. The use of DSP tasks, rather than the serial-RT task, allowed RP scheduling to maximize the extent of $\mathrm{CI}$ by ensuring frequent changes in the motor skill executed across trials (Abrahamse, Ruitenberg, de Kleine, \& Verwey, 2013). Based on the aforementioned evidence, acquisition of a novel motor sequence is expected to be facilitated following RP but not BP. It is also possible that by maximizing $\mathrm{CI}$ during the initial bout of RP, retention benefits for the new sequence will emerge.

An additional advantage of using the DSP task in the present work was the opportunity to probe the locus of any facilitation in novel skill acquisition and/or retention following training under different practice formats. Abrahamse et al. (2013) proposed that the execution of a DSP task involves three distinct planning processes. The first process, referred to as sequence initiation is reflected in the time to complete the first key-press of a DSP task. This process involves selection and preparation of the DSP task including readying its initial motor chunk. A relatively slow key-press typically observed in the middle of a DSP task, the concatenation point, indexes a cost of transitioning between motor chunks that comprise the DSP task. Finally, all other key-presses are usually executed considerably faster, often with an RT lower than $100 \mathrm{~ms}$, than those associated with the initiation and concatenation processes. This latency reflects the cost of executing the most primitive element (i.e., key-press) contained in a motor chunk. Assuming experience with prior RP contributes to improvement in a memory retrieval processes for newly learned skills, it seems reasonable to assume that any retention benefits that emerge would most likely to be observed for the sequence production processes most dependent on retrieval, that is, initiation and maybe concatenation (but see Verwey, Abrahamse, \& Eikelboom, 2010) rather than execution.

\section{Methods}

\subsection{Participants}

Participants were right-handed undergraduate students $(\mathrm{N}=36)$ that received course credit for their participation. They had no prior experience with the experimental tasks and were unaware of the specific purpose of the study. All participants completed an informed consent approved by an Institutional Review Board before any involvement in the experiment.

\subsection{Apparatus and task}

The motor skills used in the present work are characterized as discrete sequence production (DSP) tasks (Abrahamse et al., 2013). These tasks are used extensively to study motor sequence learning (Doyon et al., 2009). Each DSP task used in the present work was performed on a standard PC keyboard and involved typing a predetermined set of six key-presses in response to a visual signal that indicated the key to press. The keys used in the present experiment were the "D", "F", and "G" on which the ring, middle, and index finger of the left-hand were placed respectively and the "J", "K", and "L" keys which were associated with the index, middle, or ring fingers of the right-hand. The order of keypresses for each DSP task was dictated by the presentation of a black dot within one of the six boxes displayed horizontally across the lower third of the computer screen in a spatially compatible manner with the placement of the fingers on the keyboard (see Fig. 1A). Participants were instructed to associate the leftmost box of the display with the "D" key and press this key when a black dot appeared in this box. Alternatively, individuals were told that a black dot in the rightmost box required a press of the rightmost "L" key with the right ring finger. The black dot remained in the same location of the display until the correct key was pressed. Four unique 6-key DSP tasks were used throughout the experiment. Three of these tasks were used during random or blocked practice on Day 1 and the fourth was used as the novel task on Day 2 (see Fig. 1B).

There was a 300-2000 ms response-to-stimulus interval (RSI) after the third key press for all DSP tasks during every practice trial to encourage participants to execute each unique DSP task as two motor chunks, each containing 3 key-presses (Abrahamse et al., 2013; Verwey \& Eikelboom, 2003). Abrahamse et al. propose that the latency associated with the first key press captures the costs of organizing the sequence as a whole and initiating the first chunk whereas the time for key press four, following the longer RSI, reflects the costs related to concatenating the two motor chunks as well as initiating chunk 2 of a DSP task. The remaining key presses $(2,3,5$ and 6 in the present case) involved an execution process. All features of this experiment were programmed using E-Prime ${ }^{\circledR} 2.0$ (Psychology Software Tools, Inc., Sharpsburg, PA).

\subsection{Procedure}

Participants first read and signed an informed consent. Individuals 
A
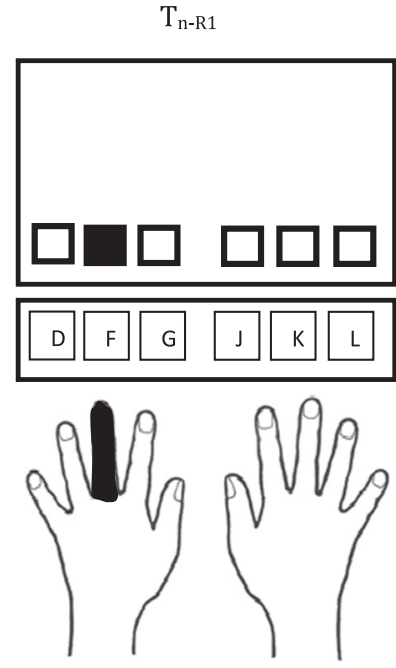

-...
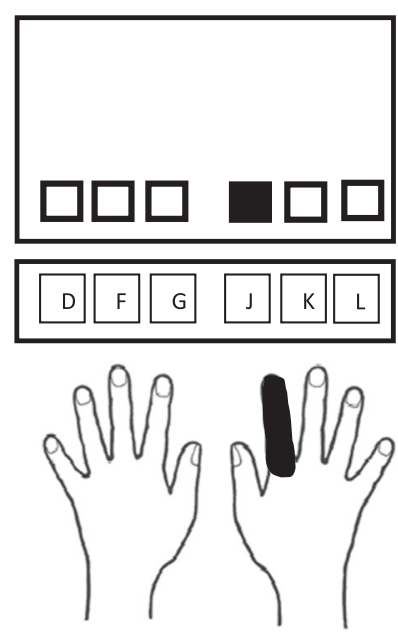

B Discrete Sequence

Production Tasks

1: K-F-G-D-J-L

2: G-L-D-K-F-J

3: L-G-J-F-K-D

4: D-J-K-G-L-F

C

BL1

\section{Block $(\mathrm{n}=4)$ \\ Block $(n=4)$ \\ Block (n=4)}

Random (n=4) 123231312 (x 11)

Random (n=4) 312123231 (x 11)

Control

\# of Trials

$111111111(x$ 11)
$222222222(x$ 11)
$333333333(x$ 11)

No Practice

99
BL2

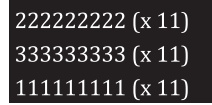

231312123 (x 11)

312123231 (x 11)

123231312 (x 11)

No Practice

BL3

BL4

Test

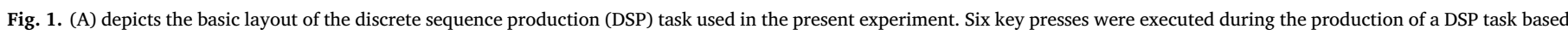

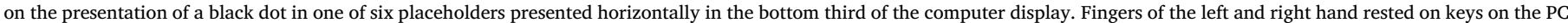

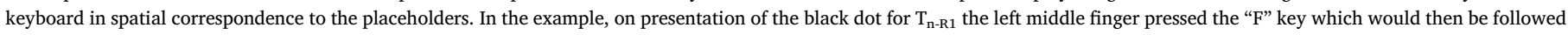

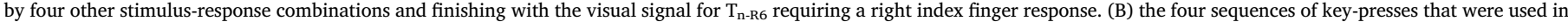

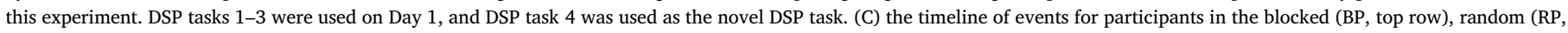

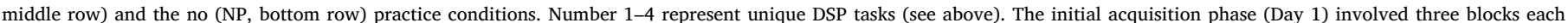

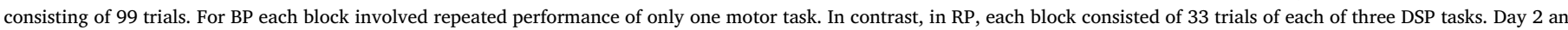

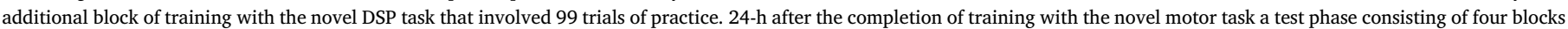

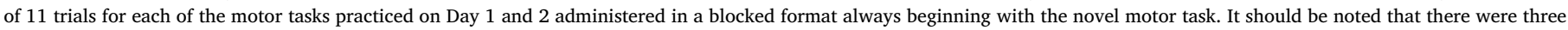
unique orders for the presentation of the DSP tasks for BP and RP to which four participants were assigned.

were assigned to one of three different practice schedule conditions, blocked (BP), random (RP), or a no (NP) practice condition (see Fig. 1C). Random practice involved a pseudo-random presentation of three of the DSP tasks during the initial acquisition phase on Day $1 .^{1}$ Individuals assigned to $\mathrm{BP}$ completed all of the assigned practice with one DSP task before experiencing any practice with another task. Approximately 24-hr after the completion of BP or RP, all participants received training with the novel DSP task. The NP condition was included to provide a baseline for acquisition and retention performance with the novel DSP task when acquired without previous RP or BP (see Fig. 1C). All participants (i.e., from the BP, RP, and NP conditions) returned to the laboratory approximately $24-\mathrm{hr}$ after the completion of practice with the novel DSP task to complete test trials for all of the DSP tasks (Kim et al., 2016).

Day 1 involved three blocks of practice each consisting of 99 trials resulting in 297 total trials of practice including 99 trials for each of three 6-key DSP tasks. During BP, all trials within a trial block (i.e., 99 trials) involved only one DSP task. For RP, each block consisted of 33 trials of each of the three separate DSP tasks. There was a 2-s interval between trials in each block. Presentation order for the sequences used during acquisition was counterbalanced. ${ }^{2}$ About 24-hr after the completion of RP or BP all participants were administered an additional block of 99 trials with a novel DSP task. Participants assigned to the NP condition only completed the block of practice with the novel DSP task (see Fig. 1C).

Performance of the novel DSP task as well as the three DSP tasks encountered during RP and BP was assessed 24-hr after practice with the novel DSP task. Thus, performance for the DSP tasks practiced in RP or BP was assessed 48-hr after original practice whereas retention for the novel DSP task occurred after only a 24-hr delay. While the focus of this work was primarily geared toward future learning (i.e., of the novel task), we still assessed retention of the original three tasks acquired in random and blocked schedules to verify that the typical contextual interference effect was observed. During the test phase, one block of 44 trials was administered beginning with 11 trials for the novel DSP tasks followed by 11 trials for each of the DSP tasks used for RP and BP

${ }^{1}$ Three RP acquisition orders were used (123,231,321, 231,321,123, and 321,123,231, where $1-3$ represented unique DSP tasks).

\footnotetext{
${ }^{2}$ Three different counterbalancing orders for BP and RP were used and are detailed in Fig. 1.
} 


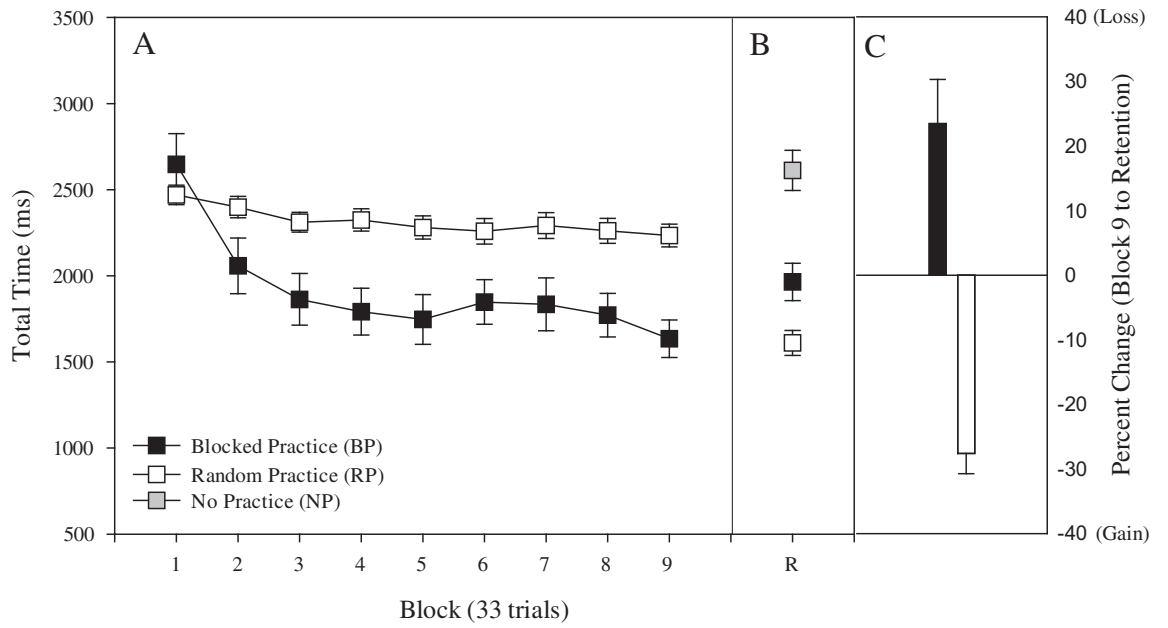

Fig. 2. (A) displays mean total time (ms) for the BP and RP conditions for the nine blocks of practice during acquisition of three DSP tasks on Day 1. (B) displays mean total time (ms) during the test block on Day 3 for the three original DSP tasks for the BP and RP conditions. (C) reveals the amount of offline improvement from the last acquisition block to the test block, indexed by percent change in Total Time, for the BP and RP conditions. Note that a positive change indicates forgetting and a negative change indicates enhancement. presented in a blocked format. ${ }^{3}$ For all tests trials, the $300-2000 \mathrm{~ms}$ RSI was removed (i.e., RSI was $0 \mathrm{~ms}$ as was all other RSIs). For all trials throughout both the acquisition and test phases, the primary dependent variable was total time (TT) which was a sum of the time for all 6-key presses executed for any one DSP task.

\section{Results}

\subsection{Acquisition and retention for the initial blocked and random practice} phase

\subsubsection{Acquisition}

For the purpose of analyzing the acquisition data, each block of 99 trials was divided into three blocks of 33 trials including 11 trials of each of the three practiced DSP tasks resulting in nine blocks of acquisition data. Mean TT for each of these blocks was calculated for each individual. Fig. 2A displays mean TT for each block of acquisition as a function of the RP and BP conditions. Mean TT for each block for each individual during acquisition was submitted to a 2 (Practice Schedule: RP, BP) $\times 9$ (Block: 1-9) analysis of variance (ANOVA) with repeated measures on the last factor. This analysis revealed significant main effects of Practice Schedule, $F(1,22)=7.52, p<0.01$, and Block, $F$ $(8176)=33.00, p<0.001$. Interpretation of these main effects was superseded by a significant Practice Schedule $\mathrm{x}$ Block interaction, $F$ $(8176)=12.90, p<0.001$. Simple main effect analyses indicated that mean TT did not differ for Block 1 and 2 as a function of practice schedule but individuals in BP exhibited significantly lower TT compared to their RP counterparts for all subsequent blocks. In addition, individuals in RP did not exhibit significant improvement in mean TT across practice during the acquisition phase. In contrast, individuals in the BP condition significantly lowered mean TT across blocks, $F(8176)$ $=7.31, p<0.001$.

\subsubsection{Retention}

Mean TT during the test block as a function of BP, RP, and NP conditions is displayed in Fig. 2B. A 3 (Practice Schedule: RP, BP, NP) between-subject ANOVA indicated that the main effect of Practice schedule was significant, $F(2,33)=25.31, p<0.001$. Post-hoc analysis revealed that the individuals that experienced RP $(M=1610 \mathrm{~ms}$, $S E M=72 \mathrm{~ms}$ ) exhibited significantly lower mean TT compared to their BP counterparts $(M=1965 \mathrm{~ms}, S E M=109 \mathrm{~ms})$ during the test trials. Mean TT for individuals assigned to BP was in turn significantly lower than observed for the individuals who only practiced the novel DSP task

\footnotetext{
${ }^{3}$ We chose to administer the test in the practice format least likely to facilitate test performance following RP (see Shea \& Morgan, 1979).
}

$(M=2612 \mathrm{~ms}, S E M=117 \mathrm{~ms})$. To further probe test performance Mean TT for the last acquisition and test blocks for each individual in the RP and BP conditions was submitted to a 2 (Practice Schedule: RP, BP) $\times 2$ (Block: last acquisition, test) ANOVA with repeated measures on the last factor. This analysis revealed a significant main effect of Block, $F(1,22)=5.61, p<0.05$. Interpretation of this main effect was superseded by a significant Practice Schedule $\times$ Block interaction, $F$ $(1,22)=59.41, p<0.001$. Simple main analyses of this interaction indicated that mean TT at the end of practice for individuals that experienced BP $(M=1634 \mathrm{~ms}, S E M=109 \mathrm{~ms})$ was significantly lower than for the RP participants $(M=2235 \mathrm{~ms}, S E M=66 \mathrm{~ms}), F(1,22)$ $=31.8, p<0.001$. This effect was reversed during the test block with mean TT for the individuals exposed to RP $(M=1610, S E M=72 \mathrm{~ms})$ being significantly lower than observed for their BP counterparts $(M=1965 \mathrm{~ms}, \quad S E M=109 \mathrm{~ms}), F(1,22)=26.78, p<0.001$. The $28 \%$ offline improvement in mean TT (i.e., change in mean TT from last acquisition to test block) for individuals with RP experience was significant, $F(1,22)=15.29, p<0.001$. In contrast, following BP, individuals exhibited a significant increase in mean TT across the retention interval, $F(1,22)=4.29, p<0.05$ (see Fig. $2 \mathrm{C}$ ).

The findings for the initial phase of the experiment were as expected and in keeping with the extant literature indicating superior acquisition performance during BP but an advantage from training in a random format at the time of test (Kim et al., 2016; Wright et al., 2016). This outcome occurred despite the retention interval being 48-h and the test trials being administered in a blocked format. The superior test performance following RP relative to BP resulted from a substantial offline improvement possibly as a result of overnight consolidation (Walker et al., 2003). In contrast, the test performance of individuals that encountered BP displayed considerable forgetting during the test trials. These data then provide the necessary foundation from which to evaluate the efficacy of recent BP and RP for future motor learning.

\subsection{Acquisition and retention of a novel motor task following previous experience with random and blocked practice}

\subsubsection{Acquisition}

For the purpose of analyzing acquisition of the novel DSP task, the block of 99 trials of practice with this task was divided into sets of 11 trials allowing calculation of mean TT for nine blocks for each individual. Fig. 3A displays mean TT as a function of the BP, RP, and NP conditions for each block of practice with the novel DSP task. These data were submitted to a 3 (Practice Schedule: RP, BP, NP) $\times 9$ (Block: 1-9) ANOVA with repeated measures on the last factor. This analysis revealed significant main effects of Practice Schedule, $F(2,33)=14.25$, $p<0.001$, and Block, $F(8264)=67.24, p<0.001$. The Practice Schedule $\times$ Block interaction was also significant, $F(16,264)=4.30$, 


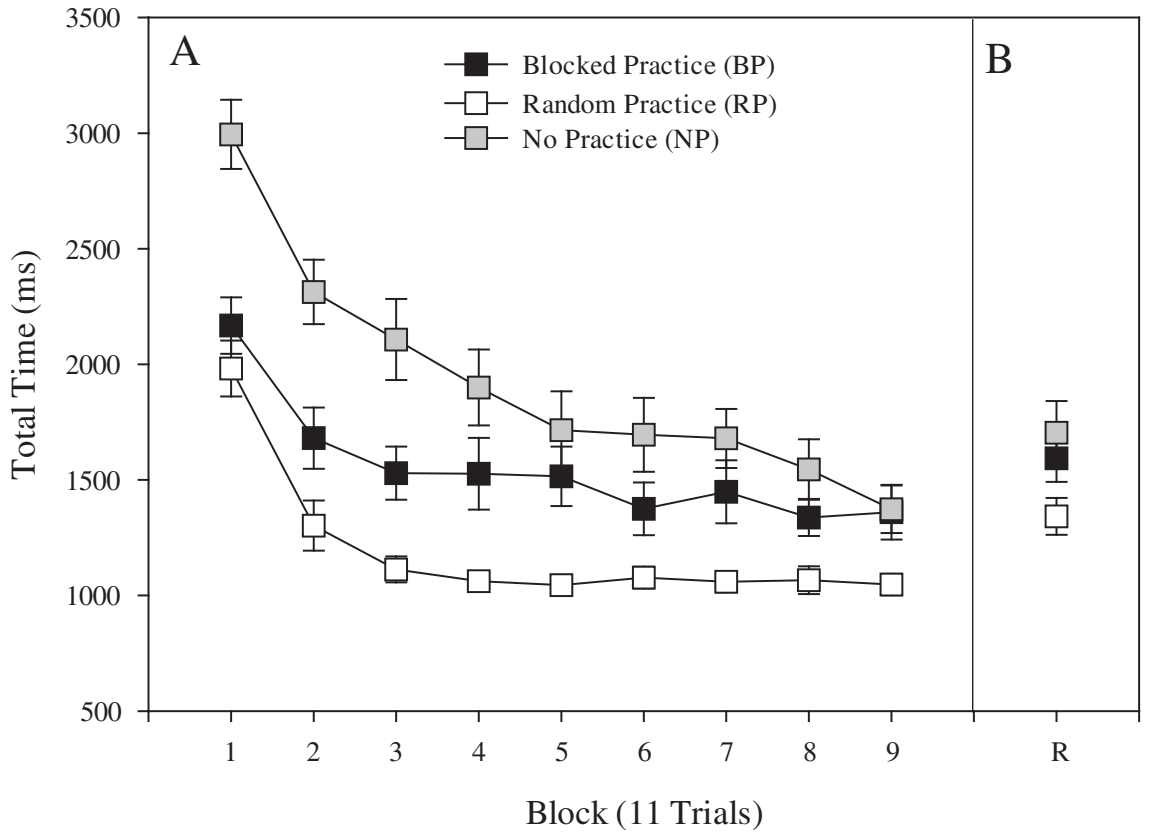

Fig. 3. (A) displays mean total time (ms) for the BP, RP, and NP conditions for the nine blocks of practice with the novel DSP task on Day 2. (B) displays mean total time (ms) during the test block on Day 3 for the novel DSP task for the BP, RP, and NP conditions. $p<0.001$. Simple main effect analysis of this interaction indicated that both BP and RP participants performed similarly for the first block of practice with the novel DSP task and significantly faster than individuals assigned to the NP condition. However, by Block 2, prior RP experience also provided a significant advantage in the form of lower mean TT compared to BP individuals. During Block 2, participants with $\mathrm{BP}$ experience continued to outperform those individuals in the NP condition. This profile continued until Block 5 at which time participants in the BP and NP conditions performed similarly and significantly poorer than their RP counterparts. However, for the final block (i.e., Block 9), while performance was still superior for RP participants, mean TT did not differ significantly as a function of practice condition.

\subsubsection{Retention}

Fig. 3B displays mean TT during the test block for the novel DSP task as a function of the BP, RP, and NP conditions. A 3 (Practice Schedule: RP, BP, NP) $\times 2$ (Block: last acquisition, test block) ANOVA with repeated measures on the last factor conducted on mean TT from the last block of acquisition and the test block. This analysis revealed a significant main effects of Practice Schedule, $F(2,33)=4.45 p<0.05$, and Block, $F(1,33)=21.35 p<0.001$ but no significant Practice Schedule $\mathrm{x}$ Block interaction, $F(2,33)=0.21, p>0.05$. Post-hoc assessment of the Practice Schedule main effect revealed significantly lower TT for participants in the RP condition $(\mathrm{M}=1195 \mathrm{~ms}$, $\mathrm{SEM}=63 \mathrm{~ms})$ compared to the $\mathrm{BP}(\mathrm{M}=1477 \mathrm{~ms}$, SEM $=110 \mathrm{~ms})$ and $\mathrm{NP}(\mathrm{M}=1539 \mathrm{~ms}, \mathrm{SEM}=121 \mathrm{~ms})$ conditions. The latter conditions did not differ significantly with respect to mean TT. This effect revealed that the lower mean TT observed at the end of acquisition for the RP compared to the BP and NP conditions remained 24-h later. Post-hoc assessment of the Block main effect revealed that mean TT at the time of test $(\mathrm{M}=1547 \mathrm{~ms}$, SEM $=107 \mathrm{~ms})$ was significantly greater than observed at the end of acquisition $(\mathrm{M}=1260 \mathrm{~ms}$, SEM $=89 \mathrm{~ms})$.

Taken together these data support the claim that the advantage of experiencing greater $\mathrm{CI}$ during motor learning extends beyond just the retention of motor sequences acquired in the initial bout of RP. In the present study, and elsewhere (Hodges et al., 2014; Kim et al., 2016), recent exposure to RP facilitates the acquisition of a new sequential behavior. This benefit emerged quite quickly, by Block 2 in the present experiment. Importantly, this performance advantage remained 24-h later at the time of test. While exposure to BP appears to offer some initial benefit when first exposed to the novel motor sequence, as evidence by lower mean TT for the first four blocks compared to the NP condition, this difference dissipated by the conclusion of practice and most critically was absent at the time of test. Thus, with respect to new learning, unlike RP, a history with BP offered no benefit to future motor learning.

\subsection{Impact of prior blocked and random practice for initiation, concatenation, and execution processes during new motor learning}

Given high CI practice supports future learning (discussed in previous result section), some additional analyses were conducted to evaluate how this advantage emerged. Recall that implementation of discrete motor sequences involves a number of independent planning processes, namely initiation, concatenation, and execution that can be isolated by using pre-structured DSP tasks such as those used in the present work (Abrahamse et al., 2013; Verwey \& Eikelboom, 2003). As noted earlier, when using a DSP task, the latency associated with the first key press captures the costs of sequence initiation referred to herein as initiation time (IT). The response time to the key press following the random non-0 RSI (key press 4) reflects the transition between two motor chunks and was termed concatenation time (CT). The remaining key presses (2, 3, 5 and 6 for DSP tasks in this experiment) each depended solely on execution demands and contributed only to execution time (ET) (Abrahamse et al., 2013).

As was the case for the earlier analyses of mean TT, the acquisition block of 99 trials of practice with the novel DSP task was divided into nine blocks of 11 trials for which mean IT, CT, and ET for each individual in the RP, BP, and NP conditions was calculated. These data were submitted to separate 3 (Practice Schedule: RP, BP, NP) $\times 9$ (Block: 1-9) ANOVA with repeated measures on the last factor. With respect to the test data, mean IT, CT, and ET for each individual for the last acquisition and test block were submitted to 3 (Practice Schedule: $\mathrm{RP}, \mathrm{BP}, \mathrm{NP}) \times 2$ (Block: last acquisition, test) ANOVA with repeated measures on the last factor. Performance for each dependent variable as a function of practice schedule during the acquisition and test blocks are displayed in Fig. 4A-F. Analyses of these data are presented in the following sections. 

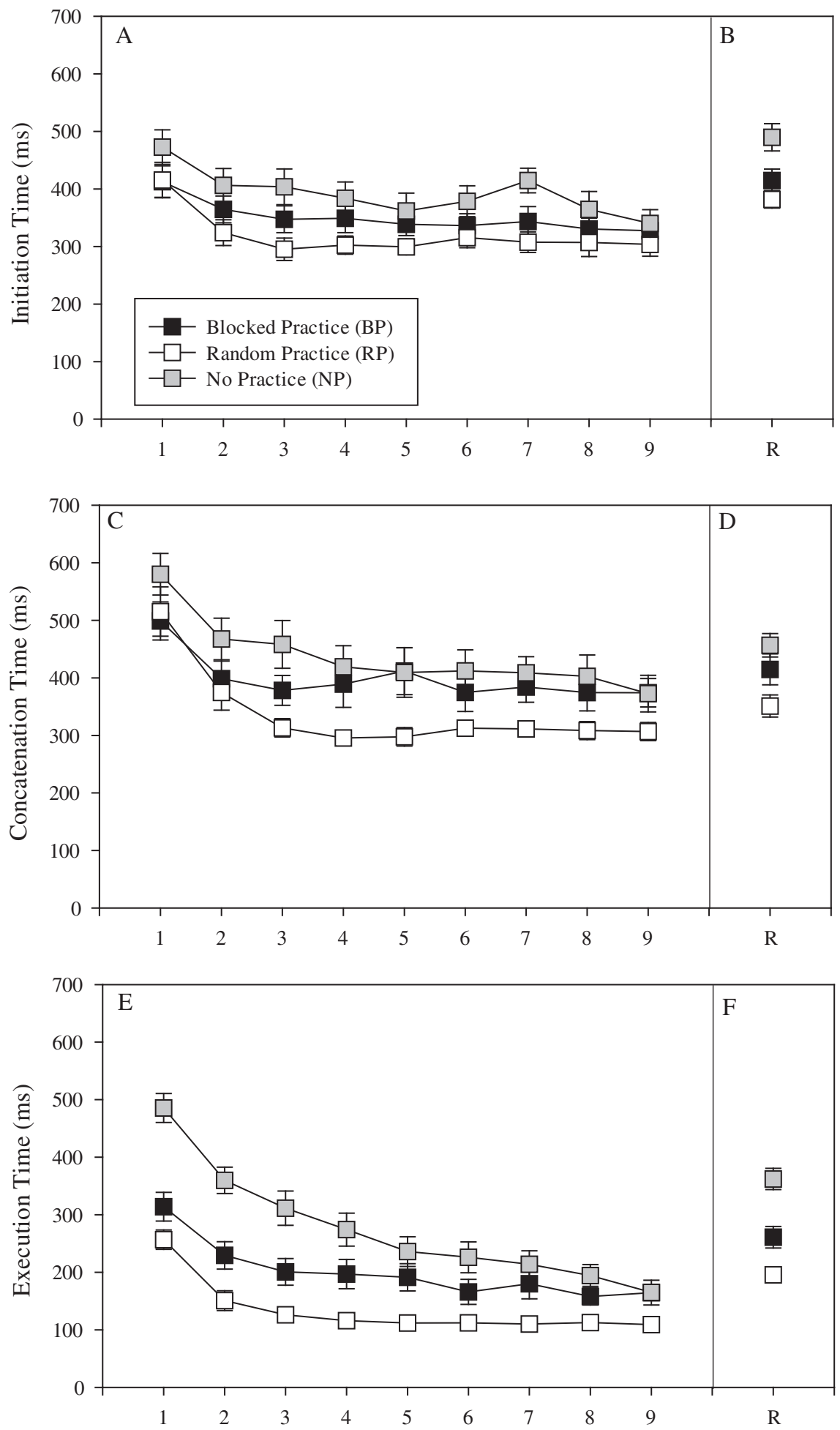

Fig. 4. (A) displays mean initiation time (ms) for the BP, RP, and NP conditions for the nine blocks of practice with the novel DSP task on Day 2. (B) displays mean initiation time (ms) during the test block on Day 3 for the novel DSP task for the BP, RP, and NP conditions. (C) displays mean concatenation time (ms) for the $\mathrm{BP}, \mathrm{RP}$, and NP conditions for the nine blocks of practice with the novel DSP task on Day 2. (D) displays mean concatenation time (ms) during the test block on Day 3 for the novel DSP task for the BP, RP, and NP conditions. (E) displays mean execution time (ms) for the BP, RP, and NP conditions for the nine blocks of practice with the novel DSP task on Day 2. (F) displays mean execution time (ms) during the test block on Day 3 for the novel DSP task for the BP, RP, and NP conditions.

Block (11 Trials)

3.4. Initiation time (IT) during the acquisition and retention of the novel DSP task

\subsubsection{Acquisition}

Fig. 4A displays mean IT as a function of BP, RP, and NP conditions for each acquisition block with the novel DSP task. Formal analysis of mean IT during acquisition revealed a significant main effect of Practice Schedule, $F(2,33)=3.31, p<0.05$, and Block, $F(8264)=14.95$. $p<0.001$. The Practice Schedule $\mathrm{x}$ Block interaction, $F(16,264)$ $=0.94, p>0.05$ was not significant. Post hoc-assessment of the significant main effect of practice schedule indicated that prior experience with RP ( $M=319 \mathrm{~ms}, S E M=20 \mathrm{~ms})$ led to significantly lower mean IT compared to those individuals with prior exposure to BP $(M=350 \mathrm{~ms}$, $S E M=23 \mathrm{~ms}$ ). Experience with BP provided an advantage, reflected in lower mean IT during acquisition, beyond that afforded by only practicing the novel DSP task $(M=392 \mathrm{~ms}, S E M=29 \mathrm{~ms})$. For all practice 
conditions, the improvement in mean IT generally occurred in the first two acquisition blocks accounting for the significant block main effect.

\subsubsection{Retention}

Fig. 4B displays mean IT during the test block as a function of RP, BP, and NP conditions. Mean IT for each individual for the last acquisition and test block were submitted to 3 (Practice Schedule: RP, BP, NP) $\times 2$ (Block: last acquisition, test) ANOVA with repeated measures on the last factor. This analysis revealed significant main effects of Practice Schedule, $F(2,33)=3.42, p<0.05$ and Block, $F(1,33)$ $=116.81, p<0.001$. Interpretation of these significant main effects was superseded by the significant Practice Schedule $\mathrm{x}$ Block interaction, $F(2,33)=5.34, p<0.01$. Simple main effect analyses of this interaction revealed similar mean IT at the conclusion of practice for individuals assigned to RP ( $\mathrm{M}=304 \mathrm{~ms}$, SEM $21 \mathrm{~ms})$, BP $(M=327 \mathrm{~ms}$, $S E M=23 \mathrm{~ms})$ and $\mathrm{NP}(M=340 \mathrm{~ms}, S E M=24 \mathrm{~ms}), F(2,33)=0.44$, $p>0.05$. At the time of test, the mean IT for the NP condition ( $M=490 \mathrm{~ms}, S E M=24 \mathrm{~ms}$ ) was significantly greater than that observed for the RP $(M=382 \mathrm{~ms}, S E M=15 \mathrm{~ms})$ and BP $(M=415 \mathrm{~ms}$, $S E M=20 \mathrm{~ms}$ ) conditions. Mean IT for the latter conditions did not differ significantly from one another. Simple main effect analysis also indicated that mean IT increased significantly from the end of acquisition to the test block for both the NP, $F(1,33)=14.56, p<0.01$ and BP, $F(1,33)=4.97, p<0.05$, conditions. In contrast, the mean IT for individuals with RP experience did not change significantly across the 24-h delay, $F(1,33)=3.96, p>0.05$.

\subsection{Concatenation Time (CT) during the acquisition and retention of a novel DSP task}

\subsubsection{Acquisition}

Fig. 4C displays mean CT as a function of BP, RP, and NP conditions for each acquisition block with the novel DSP task. The analysis of mean CT during the acquisition phase revealed significant main effects of Practice Schedule, $F(2,33)=4.37, p<0.05$, and Block, $F(8264)$ $=21.83 . p<0.001$. This analysis failed to reveal a significant interaction of Practice Schedule $\times$ Block, $F(16,264)=1.22, p>0.05$. Interpretation of the significant main effect of practice schedule indicated that mean CT during acquisition was significantly lower for the RP condition $(M=337 \mathrm{~ms}, \quad S E M=19 \mathrm{~ms})$ than observed for BP ( $M=398 \mathrm{~ms}, S E M=32 \mathrm{~ms})$. Moreover, the mean CT for the NP condition $(M=437 \mathrm{~ms}, S E M=36 \mathrm{~ms})$ was significantly higher than for the other practice conditions. As expected, the main effect of Block was a result of a reduction in mean CT across practice for all practice conditions with the largest improvements occurring during the initial three blocks.

\subsubsection{Retention}

Fig. 4D displays mean CT for individuals in the BP, RP, or NP conditions when performing the novel DSP task during the test block. Mean CT from the last acquisition and test blocks were submitted to a 3 (Practice Schedule: RP, BP, NP) $\times 2$ (Block: last acquisition, test block) ANOVA with repeated measures on the last factor. This analysis revealed significant main effects of Practice Schedule, $F(2,33)=5.23$, $p \leq 0.01$, and Block, $F(1,33)=13.18, p<0.001$ but failed to reveal a Practice Schedule $\mathrm{x}$ Block interaction, $F(2,33)=0.82, p>0.05$. Subsequent post-hoc analyses of the main effect of Practice Schedule indicated that mean CT for RP individuals $(M=329 \mathrm{~ms}$, $S E M=17 \mathrm{~ms})$ was significantly lower than for their BP $(M=395 \mathrm{~ms}, S E M=26 \mathrm{~ms})$ and NP ( $M=415 \mathrm{~ms}, S E M=26 \mathrm{~ms})$ counterparts. Performance for the latter conditions did not differ significantly. Moreover, Mean CT increased from the end of acquisition $(M=351 \mathrm{~ms}, S E M=24 \mathrm{~ms})$ to the test trials $(M=408, S E M=22 \mathrm{~ms})$ accounting for the significant Block main effect.
3.6. Execution Time (ET) during the acquisition and retention of a novel DSP task

\subsubsection{Acquisition}

Fig. 4E displays mean ET as a function of BP, RP, and NP conditions for each acquisition block with the novel DSP task. Analysis of these data revealed significant main effects of Practice Schedule, $F(2,33)$ $=17.61, p<0.001$, and Block, $F(8264)=76.88, p<0.001$. Interpretation of these main effects was superseded by significant Practice Schedule $\times$ Block interaction, $F(16,264)=6.86, p<0.001$. Simple main effect analysis revealed an early advantage in mean ET for individuals in the RP and BP compared to the NP conditions. However, by Block 5 mean ET for the BP condition and NP conditions was not significantly different.

\subsubsection{Retention}

Fig. 4F displays mean ET for the BP, RP, or NP conditions when performing the novel DSP task during the test block. Mean ET from the last block of acquisition and the test block for each individuals was submitted to a 3 (Practice Schedule: RP, BP, NP) $\times 2$ (Block: last acquisition, test block) ANOVA with repeated measures on the last factor. This analysis revealed significant main effects of Practice Schedule, $F$ $(2,33)=14.96, p<0.001$, and Block $F(1,33)=204.49, p<0.001$. Interpretation of these main effect was superseded by a significant Practice Schedule $\times$ Block interaction, $F(2,33)=15.79, p<0.001$. Simple main effect analysis indicated that mean ET for individuals in the RP $(M=109 \mathrm{~ms}, S E M=9 \mathrm{~ms})$, BP $(M=165 \mathrm{~ms}, S E M=22 \mathrm{~ms})$, and NP $(M=165 \mathrm{~ms}, S E M=14 \mathrm{~ms})$ conditions did not differ significantly at the end of acquisition, $F(2,33)=0.91, p>0.05$. However, at the time of test, participants in the RP condition ( $M=196 \mathrm{~ms}$, $S E M=13 \mathrm{~ms}$ ) exhibited significantly lower mean ET compared to the BP condition $(M=261 \mathrm{~ms}, S E M=19 \mathrm{~ms})$ who in turn revealed a lower ET than observed for the NP condition $(M=362 \mathrm{~ms}$, $S E M=18 \mathrm{~ms}), F(2,33)=6.15, p<0.05$. Simple main effect analysis also revealed that mean ET increased for all practice schedule conditions from the end of acquisition to the test trials, however the increase was greater for the NP condition, $F(1,33)=46.7, p<0.01$, compared to the BP, $F(1,33)=11.16, p<0.01$, and RP conditions, $F(1,33)$ $=8.99, p<0.01$.

Taken as a whole, these data revealed that prior experience with RP and BP provides an immediate and broad advantage in motor planning during the acquisition of a novel DSP task. This advantage during practice was short-lived for individuals with BP experience with only the RP participants continuing to exhibit superior sequence performance especially with respect to concatenating motor chunks (i.e., CT) and executing each element within each motor chunk (i.e., ET) in the later stages of acquisition. While all participants exhibit some forgetting across the retention interval, the severity of the loss was significantly less for individuals with previous RP. Prior BP experience was mostly ineffective at reducing the extent of forgetting as evidenced by retention losses that were similar to that observed for individuals in the NP condition.

\section{Discussion}

The present study was, at least in part, designed to examine a potential shortcoming in earlier work by Kim et al. (2016) that may have limited the extent of and the resultant effectiveness of prior RP experience for future learning (see also, Hodges et al., 2014). Specifically, the use of a serial RT task by Kim et al. inadvertently created a modified $\mathrm{BP}$ schedule thus reducing the degree of $\mathrm{CI}$ created in their RP condition. In the present case, the use of a DSP task afforded the opportunity to maximize the $\mathrm{CI}$ induced during random training and examined the impact of extent of $\mathrm{CI}$ in prior training on the acquisition and retention of a novel motor skill. 


\subsection{RP experience facilitates acquisition and retention of future motor} learning

As anticipated, consistent with findings of Hodges et al. (2014) and Kim et al. (2016), the performance facilitation from prior RP on the acquisition of a novel skill, was quite robust. A clear performance advantage when practicing the novel DSP task emerged for the individuals with RP experience compared to those with some BP experience or individuals that only practiced the novel DSP task. This benefit emerged almost immediately and was broad in scope. While the expectation was that gains would be observed for "retrieval-related" planning activity, namely sequence initiation and concatenation, there was clear evidence that the RP benefit also surfaced for execution processes (Abrahamse et al., 2013). It has been proposed that a primary consequence of RP experience is the integration of new motor memories into an elaborate memory network that affords easy access to the new knowledge (Lin et al., 2013; Shea \& Zimny, 1988; Wright et al., 2016). It seems reasonable then that subsequent learning, particularly that involving the acquisition of a similar motor skills, should benefit from the existence of such a memory network. The acquisition benefits for the novel task reported herein are consistent with this account. Indeed, poor acquisition performance, characteristic of RP frequently reported in previous studies addressing CI and motor learning, did not occur during the acquisition phase with the novel DSP task in the present study (Brady, 2004; Magill \& Hall, 1990; Shea \& Zimny, 1983; Wright et al., 2016). Rather, these individuals exhibited very efficient encoding of the novel skill resulting from an enhancement of retrieval and execution processes.

The effective use of a wide array of preparatory process (i.e., initiation, concatenation, execution) by RP noted in this study is consistent with previous reports that revealed recruitment of a broad network of neural regions, critical for motor skill acquisition, during RP (Dayan \& Cohen, 2011; Doyon et al., 2009; Floyer-Lea \& Matthews, 2005; Hardwick, Rottschy, Miall, \& Eickhoff, 2013; Penhune \& Steele, 2012). This is in contrast to activating the default network, as is the case following BP, which occurs during undisturbed thinking or when not engaged in evaluation of the external world (Buckner, AndrewsHanna, \& Schacter, 2008). Indeed, individuals exposed to RP typically exhibit a rapid reduction in use of the default network early in practice focusing instead on using neural resources important for planning motor responses and establishing motor memories (Cross, Schmitt, \& Grafton, 2007; Wymbs \& Grafton, 2009).

A novel finding in the present study was the persistence of this benefit from acquisition to retention of the novel motor skill for RP experienced individuals. Previous work failed to demonstrate any advantage of a history with RP beyond initial encoding that is inferred from performance during acquisition (Hodges et al., 2014; Kim et al., 2016). The general benefit at retention (i.e., mean TT) resulted from contributions from all processes, described by Abrahamse and colleagues (Abrahamse et al., 2013), central to successful implementation of a sequential behavior. In the case of concatenation and execution, the greater relative improvement in these processes during initial encoding compared to BP subjects was also maintained across the retention interval despite some forgetting. Thus, while post-practice processing (e.g., consolidation) plays an important role during the original bout of RP (see Fig. 2C), the gains from RP experience for new learning appear to result from rapid encoding of new information that is more resilient to forgetting but not enhanced offline due to consolidation.

In contrast, for initiation, facilitation from prior RP also led to a reduced forgetting rate compared to individuals that were privy to $\mathrm{BP}$ or NP. For the initiation process then the existence of a benefit from training history appears more latent than the other processes relying in part more heavily on the involvement of memory-formation processes that occur after the completion of practice. Why the initiation process responds differently to training history, or more specifically lacks a more robust influence of prior RP, is difficult to determine from the present data. Given the concatenation was facilitated almost immediately for the RP-experienced participants this difference is unlikely related to retrieval of motor chunks as both processes (i.e., initiation and concatenation) have this feature in common. It is important to note that initiation, as indexed by the latency associated with first key press in the DSP task, probably involves a myriad of other preparatory events when performing sequential behaviors beyond just retrieval-related events (Abrahamse et al., 2013). It is not difficult to imagine then that some other, as yet undefined planning process, is susceptible to training history that interact with post-practice processes when implemented. Clearly, this issue needs additional experimental scrutiny to be completely understood. Despite the minor lack of clarity regarding prior RP experience for sequence initiation, one cannot fail to see the positive influence of RP experience for future motor learning. These data, particularly those from the retention phase, point to the importance of maximizing CI during periods of practice to fully capitalize on the efficacy of the random practice format for learning in general (cf., Kim et al., 2016).

\subsection{Related practice experience, even presented in a blocked format, can offer limited benefits for future motor learning}

In the previous section focus was on the utility of maximizing CI during training to support future learning. Beyond this, it also seems reasonable to assume that any prior experience with similar motor skills, even if not presented in a random format, should be advantageous for later learning compared to no prior experience. This is of course the basic assumption of proactive facilitation or transfer. Congruent with this notion was the finding that BP experience enhanced early acquisition efforts compared to the individuals in the NP condition but these benefits dissipated by the end of training with the new motor task. This was true for all planning processes, namely initiation, concatenation, and execution. Nonetheless, BP experience offered protection from forgetting when compared to only practicing the novel DSP task particularly in the case of initiation and execution. That is, performance loss across the 24-h retention interval was less for individuals with a history of BP rather than no prior training with similar motor sequences. Thus, practice experience per se. doesn't change the manner in which encoding unfolds but the resultant motor memories developed by individuals with such experience are more resilient to forgetting.

The formation of motor chunks has been described as a fundamental strategy used to facilitate early improvement in the performance of complex serial behaviors and central to motor skill learning (Sakai, Kitaguchi, \& Hikosaka, 2003; Verwey \& Eikelboom, 2003). According to Abrahamse et al. (2013) the implementation of smooth transitions between motor chunks is reflected in CT. A history with BP offered little benefit, beyond that obtained merely through practicing the novel DSP, for this process. This finding is congruent with a previous report in which individuals exposed to BP restructured a 4-element timing task into a unitary response (i.e., exhibited evidence of use of concatenation to enhance sequence implementation) early in practice in a manner also observed for individuals in RP. However, following a 24-h delay, while individuals assigned to RP continued to execute the movement sequence as a single unit, the participants trained in a blocked schedule returned to treating the practiced motor sequence as if it consisted of numerous discrete components. These data exposed the transitory use of the concatenation process during BP as means of supporting successful sequence production (Wright et al., 2004). Indeed, Wright et al. (2016) recently proposed that two neural regions, dorsal premotor area and the SMA complex are recruited earlier and more extensively during a high but not a low CI practice environment. Critically, activation of these regions have been reported to be central to establishing a memory structure amenable to long-term storage and have important roles in transitioning between movement primitives that constitute complex motor behaviors (Hardwick et al., 2013; Kennerley, Sakai, \& 
Rushworth, 2004; Nachev, Kennard, \& Husain, 2008; Rushworth, Walton, Kennerley, \& Bannerman, 2004).

\subsection{Unique processes promoted from increased contextual interference during practice influence both proximal and distal motor learning}

The primary focus of the present work was evaluating if recent experience with a high CI practice format facilitates future learning. It is important however to not overlook the robust learning benefits observed during the initial period of RP training, As expected, the outcome from the initial bout of BP and RP revealed that experiencing greater (i.e., RP) rather than less interference (i.e., BP), disrupted initial performance during training but supported superior delayed retention (Brady, 2004; Shea \& Morgan, 1979; Wright et al., 2016). In the present study this profile emerged despite the test interval being relatively long compared to many other studies (i.e., 48-h, although see Lin et al., $2013)^{4}$ as well as the test being administered in an incongruent test environment (i.e., blocked format) for the RP individuals. These data speak to the robustness of this learning effect.

Interestingly, the advantage gained from RP in this case was entirely a product of performance enhancement occurring during the interval between the end of practice and the test. This post-practice period, assumed to be the temporal locus of consolidation, has been the subject of a great deal of research in recent years with respect to its importance to the formation of resilient memories. Indeed, the development of motor memories can been inhibited with alternative task practice (Rhee et al., 2016) or the application of non-invasive brain stimulation (Muellbacher, Ziemann, Boroojerdi, Cohen, \& Hallett, 2001) during this interval. In contrast, affording sleep (Born, Rasch, \& Gais, 2006; Walker et al., 2003) or exercise (Rhee et al., 2016; Roig, Skriver, LundbyeJensen, Kiens, \& Nielsen, 2012; Skriver et al., 2014) during this timeframe has been reported to exerts a positive impact on memory development. The present findings, as well as others (Robertson, PascualLeone, \& Miall, 2004), suggest that the schedule of practice is an additional factor that can influence consolidation engaged by the learner at the conclusion of practice. At this point it cannot be determined if the practice schedule per se has a direct influence on consolidation or if this feature of the training environment interacts with processes induced during sleep. Clearly, in future experimental endeavors, the direct impact of CI on consolidation needs to be isolated by using a retention interval that does not include sleep.

There was a striking difference in the way that RP exposure influenced the retention of the novel DSP task compared to the motor skills learned during the original bout of RP. While the latter, as just described, was a result of effective post-practice processing during the 48$\mathrm{h}$ retention interval, prior RP experience did not provide any enhancement for the newly acquired motor memory. Rather the relative benefit was manifest as a slower rate of forgetting compared to their BP counterparts. Thus, RP appears to contribute to memory development in unique ways. In one case, memories for multiple skills practiced together are elaborated offline if given sufficient time to be consolidated (see Experiment 1 in Kim et al., 2016). On the other hand, a mere past history with RP isn't sufficient to take advantage of postpractice consolidation to the extent necessary to observe offline enhancement but can lead to the development of a more resilient motor memory following practice of a new skill. A more effective implementation of a broad set of key motor planning processes resulting from recent RP make an important contribution to the emergence of this outcome.

In summary, an extraordinary number of studies in the last forty years have highlighted the efficacy of experiencing more CI in training

\footnotetext{
${ }^{4}$ Most studies addressing contextual interference and motor learning use a 24-h interval between the training and test phases. However there are studies that have used more immediate tests (i.e., in the order of a few minutes) and longer intervals (e.g., 72 -hrs in Lin et al., 2013)
}

environments when learning multiple skills (Magill \& Hall, 1990; Shea \& Morgan, 1979; Wright et al., 2016). However, these efforts has focused exclusively on the resultant performance of the motor skills practiced in the original bout of practice. The important contribution of the present work is the demonstration that a history with high CI practice can influence learning of skills not contained in the original bout of RP. This appears to be accomplished by encouraging a more effective use of motor planning processes that are central features of a contemporary theoretical account for the learning of discrete motor skills (Abrahamse et al., 2013).

\section{References}

Abrahamse, E. L., Ruitenberg, M. F. L., de Kleine, E., \& Verwey, W. B. (2013). Control of automated behavior: Insights from the discrete sequence production task. Frontiers in Human Neuroscience, 7.

Adams, S. G., \& Page, A. D. (2000). Effects of selected practice and feedback variables on speech motor learning. Journal of Medical Speech-Language Pathology, 8(4), 215-220.

Born, J., Rasch, B., \& Gais, S. (2006). Sleep to remember. The Neuroscientist, 12(5), 410-424. http://dx.doi.org/10.1177/1073858406292647.

Brady, F. (2004). Contextual interference: A meta-analytic study. Perceptual and Motor Skills, 99(1), 116-126.

Buckner, R. L., Andrews-Hanna, J. R., \& Schacter, D. L. (2008). The brain's default network - anatomy, function, and relevance to disease. Year in cognitive neuroscience. 1124. Year in cognitive neuroscience (pp. 1-38).

Cross, E. S., Schmitt, P. J., \& Grafton, S. T. (2007). Neural substrates of contextual interference during motor learning support a model of active preparation. Journal of Cognitive Neuroscience, 19(11), 1854-1871.

Dayan, E., \& Cohen, L. G. (2011). Neuroplasticity subserving motor skill learning. Neuron, 72(3), 443-454. http://dx.doi.org/10.1016/j.neuron.2011.10.008.

Doyon, J., Bellec, P., Amsel, R., Penhune, V., Monchi, O., Carrier, J., ... Benali, H. (2009). Contributions of the basal ganglia and functionally related brain structures to motor learning. Behavioural Brain Research, 199(1), 61-75. http://dx.doi.org/10.1016/j. bbr.2008.11.012.

Floyer-Lea, A., \& Matthews, P. M. (2005). Distinguishable brain activation networks for short- and long-term motor skill learning. Journal of Neurophysiology, 94(1), 512-518. http://dx.doi.org/10.1152/jn.00717.2004.

Goode, S., \& Magill, R. A. (1986). Contextual interference effects in learning 3 badminton serves. Research Quarterly for Exercise and Sport, 57(4), 308-314.

Hanlon, R. E. (1996). Motor learning following unilateral stroke. Archives of Physical Medicine and Rehabilitation, 77(8), 811-815.

Hardwick, R. M., Rottschy, C., Miall, R. C., \& Eickhoff, S. B. (2013). A quantitative metaanalysis and review of motor learning in the human brain. NeuroImage, 67, 283-297.

Hodges, N. J., Lohse, K. R., Wilson, A., Lim, S. B., \& Mulligan, D. (2014). Exploring the dynamic nature of contextual interference: Previous experience affects current practice but not learning. Journal of Motor Behavior, 46(6), 455-467.

Kennerley, S. W., Sakai, K., \& Rushworth, M. F. S. (2004). Organization of action sequences and the role of the pre-SMA. Journal of Neurophysiology, 91(2), 978-993. http://dx.doi.org/10.1152/jn.00651.2003.

Kim, T., Rhee, J., \& Wright, D. L. (2016). Allowing time to consolidate knowledge gained through random practice facilitates later novel motor sequence acquisition. Acta Psychologica, 163, 153-166.

Knock, T. R., Ballard, K. J., Robin, D. A., \& Schmidt, R. A. (2000). Influence of order of stimulus presentation on speech motor learning: A principled approach to treatment for apraxia of speech. Aphasiology, 14(5-6), 653-668.

Lin, C. H., Chiang, M. C., Knowlton, B. J., Iacoboni, M., Udompholkul, P., \& Wu, A. D. (2013). Interleaved practice enhances skill learning and the functional connectivity of fronto-parietal networks. Human Brain Mapping, 34(7), 1542-1558.

Magill, R. A., \& Hall, K. G. (1990). A review of the contextual interference effect in motor skill acquisition. Human Movement Science, 9(3-5), 241-289.

Muellbacher, W., Ziemann, U., Boroojerdi, B., Cohen, L., \& Hallett, M. (2001). Role of the human motor cortex in rapid motor learning. Experimental Brain Research, 136(4), 431-438.

Nachev, P., Kennard, C., \& Husain, M. (2008). Functional role of the supplementary and pre-supplementary motor areas. Nature Reviews Neuroscience, 9(11), 856-869. http:// dx.doi.org/10.1038/Nrn2478.

Ollis, S., Button, C., \& Fairweather, M. (2005). The influence of professional expertise and task complexity upon the potency of the contextual interference effect. Acta Psychologica, 118(3), 229-244.

Penhune, V. B., \& Steele, C. J. (2012). Parallel contributions of cerebellar, striatal and M1 mechanisms to motor sequence learning. Behavioural Brain Research, 226(2), 579-591. http://dx.doi.org/10.1016/j.bbr.2011.09.044.

Rhee, J., Chen, J., Riechman, S. M., Handa, A., Bhatia, S., \& Wright, D. L. (2016). An acute bout of aerobic exercise can protect immediate offline motor sequence gains. Psychological Research Psychologische Forschung, 80(4), 518-531.

Robertson, E. M., Pascual-Leone, A., \& Miall, R. C. (2004). Current concepts in procedural consolidation. Nature Reviews Neuroscience, 5(7), 576-582.

Roig, M., Skriver, K., Lundbye-Jensen, J., Kiens, B., \& Nielsen, J. B. (2012). A single bout of exercise improves motor memory. PLoS One, 7(9).

Rushworth, M. F. S., Walton, M. E., Kennerley, S. W., \& Bannerman, D. M. (2004). Action sets and decisions in the medial frontal cortex. Trends in Cognitive Sciences, 8(9), 410-417. http://dx.doi.org/10.1016/j.tics.2004.07.009. 
Sakai, K., Kitaguchi, K., \& Hikosaka, O. (2003). Chunking during human visuomotor sequence learning. Experimental Brain Research, 152(2), 229-242.

Schneider, V. I., Healy, A. F., Bourne, L. E., Jr., \& Bourne, L. E., Jr. (1998). Contexual interference effects in foreign language vocabulary acquisition and retention. In A. F. Healy (Ed.). Foreign language learning: Psycholinguistic studies on training and retention (pp. 77-90). Mahwah, NJ, USA: Lawrence Erlbaum Associates, Inc., Publishers.

Shea, J. B., \& Morgan, R. L. (1979). Contextual interference effects on the acquisition, retention, and transfer of a motor skill. Journal of Experimental Psychology: Human Learning and Memory, 5(2), 179-187.

Shea, J. B., \& Zimny, S. T. (1983). Context effects in memory and learning. In R. Magill (Ed.). Memory and control of action (pp. 145-166). (Amsterdam: North Holland).

Shea, J. B., \& Zimny, S. T. (1988). Knowledge incorporation in motor representation. In O. J. Meijer, \& J. Roth (Eds.). Complex movement behaviour (pp. 345-366). (Amsterdam: North Holland).

Skriver, K., Roig, M., Lundbye-Jensen, J., Pingel, J., Helge, J. W., Kiens, B., \& Nielsen, J. B. (2014). Acute exercise improves motor memory: Exploring potential biomarkers. Neurobiology of Learning and Memory, 116, 46-58.

Smith, P. J. K., \& Davies, M. (1995). Applying contextual interference to the Pawlata roll. Journal of Sports Sciences, 13(6), 455-462.

Verwey, W. B., \& Eikelboom, T. (2003). Evidence for lasting sequence segmentation in the discrete sequence-production task. Journal of Motor Behavior, 35(2), 171-181.
Verwey, W. B., Abrahamse, E. L., \& De Kleine, E. (2010). Cognitive processing in new and practiced discrete keying sequences. Frontiers in Psychology, 1, 32. http://dx.doi.org/ 10.3389/fpsyg.2010.00032.

Walker, M. P., Brakefield, T., Hobson, J. A., \& Stickgold, R. (2003). Dissociable stages of human memory consolidation and reconsolidation. Nature, 425(6958), 616-620.

Wright, D. L., Brueckner, S., Black, C. B., Magnuson, C., \& Immink, M. A. (2004). Longterm motor programming improvements occur via concatenation of movement sequences during random but not during blocked practice. Journal of Motor Behavior, 36(1), 39-50.

Wright, D. L., Verwey, W., Buchanen, J., Chen, J., Rhee, J., \& Immink, M. (2016). Consolidating behavioral and neurophysiologic findings to explain the influence of contextual interference during motor sequence learning. Psychonomic Bulletin \& Review, 23(1), 1-21.

Wymbs, N. F., \& Grafton, S. T. (2009). Neural substrates of practice structure that support future off-line learning. Journal of Neurophysiology, 102(4), 2462-2476.

Yang, H. O., Li, C. H., \& Chiang, M. C. (2014). Using group independent component analysis to investigate resting-state functional networks underlying motor sequence learning. In F. C. Su, S. H. Wang, \& M. L. Yeh (Eds.). 1st global conference on biomedical engineering \& 9th Asian-Pacific conference on medical and biological engineering (pp. 120-123). Springer International Publishing. 